\title{
Abundances of recently discovered planetary nebulae towards the galactic bulge ${ }^{\star \star \star \star}$
}

\author{
A. V. Escudero and R. D. D. Costa \\ Departamento de Astronomia, IAG/USP, CP 3386, 01060-970 São Paulo, Brasil \\ e-mail: escudero@iagusp.usp.br; roberto@iagusp.usp.br
}

Received 30 April 2001 / Accepted 24 September 2001

\begin{abstract}
In this work we report spectrophotometric observations of a planetary nebula sample towards the galactic bulge. A total of $45 \mathrm{PNe}$ was observed and their physical parameters (electron density and temperature) were derived. Ionic abundances were calculated using a three-level atom model and abundances were derived through ionisation correction factors. Results show low abundance objects at high galactic latitudes, indicating a possible vertical gradient inside the bulge. A few objects with low N/O ratio were found, which could have originated from old, low mass progenitors.
\end{abstract}

Key words. ISM: planetary nebulae - galactic bulge - techniques: spectroscopic

\section{Introduction}

The galactic bulge is a structure whose properties have been extensively studied in recent years. In particular, abundance studies show many aspects of bulge populations, sometimes contradictory: while earlier works (e.g. Rich 1988) indicate abundances up to $[\mathrm{Fe} / \mathrm{H}] \sim 0.30$, other more recent studies (McWilliam \& Rich 1994; Ibata \& Gilmore 1995) indicate subsolar values, derived from $\mathrm{K}$ giants. Using spectra of integrated light from the galactic bulge in Baade's window, Idiart et al. (1996) derive a mean metallicity $[\mathrm{Fe} / \mathrm{H}]=-0.02$, which is consistent with the mean values found by Rich (1988) and McWilliam \& Rich (1994). Comprehensive revisions of abundance data and the current ideas on Galaxy evolution can be found in van den Bergh (1996) and McWilliam (1997).

In this context, abundances from planetary nebulae $(\mathrm{PNe})$ play an important role. The derivation of nebular abundances of light elements like $\mathrm{O}, \mathrm{S}$, Ar from the flux of forbidden emission lines is quite convenient at the large distances and strong reddening of the galactic bulge.

\footnotetext{
Send offprint requests to: R. D. D. Costa,

e-mail: roberto@iagusp.usp.br

* Based on observations made at the European Southern Observatory (Chile) and Laboratório Nacional de Astrofísica (Brasil).

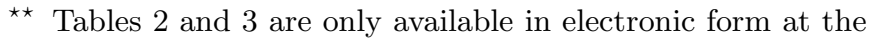
CDS via anonymous ftp to

cdsarc.u-strasbg.fr $(130.79 .128 .5)$ or via

http://cdsweb.u-strasbg.fr/cgi-bin/qcat?J/A+A/380/300
}

Abundances from these objects reflect the state of the interstellar medium at the progenitor formation epoch, and therefore can be related directly to stellar abundances. On the other hand, He and $\mathrm{N}$ abundances are modified along the stellar evolution of the progenitor and can be related to their masses (and age).

Some previous works (Ratag et al. 1992, 1997; Cuisinier et al. 2000) give abundances of different samples of bulge PNe. In both cases, objects were selected from the Acker et al. (1992) catalogue, and results indicate an abundance pattern comparable to their disk counterparts. A point yet to be tackled is the possibility of the existence of a chemical abundance gradient within the bulge. There are several suggestions for the existence of a chemical abundance gradient within the bulge, as proposed by Minitti et al. (1995), Frogel et al. (1999) or Tiede et al. (1995). In this last work they found evidence of a gradient in $\mathrm{Fe} / \mathrm{H}$ for the region $-12 \leq b \leq-3$.

As part of a long-term program of derivation of nebular abundances, we have carried out observations of southern planetary nebulae (see, e.g. Costa et al. 1996, 2000 and references therein) aiming to derive their nebular physical parameters and chemical abundances. In this work, we report spectroscopic observations for a sample of $44 \mathrm{PNe}$ towards the galactic bulge. From this sample, chemical abundances were derived. Objects were selected from new samples recently published (Kohoutek 1994; Beaulieu et al. 1999), and with these results we intend to enlarge the sample of bulge PNe with known chemical abundances, to obtain the abundance distribution of the intermediate mass star 
population in the galactic bulge. These results give important clues for the chemical enrichment and evolution of the galactic bulge.

In Sect. 2 we present our observation and reduction procedures, in Sect. 3 physical parameters and chemical abundances derivation are derived, in Sect. 4 results are discussed and general conclusions are drawn.

\section{Observations and data reduction}

\subsection{Observations}

Observation were made at the $1.60 \mathrm{~m}$ telescope of the National Laboratory for Astrophysics (LNA, Brasópolis, Brazil) and at the $1.52 \mathrm{~m}$ of the European Southern Observatory (ESO/La Silla, Chile). In both telescopes, Cassegrain Boller \& Chivens spectrographs were used, with reciprocal dispersions of $4.4 \AA /$ pixel at LNA and $2.2 \AA /$ pixel at ESO. A long east-west slit was used in all observations. In both observatories, slits of 2 arcsecs width were used. The $\log$ of the observations and identification of the objects are given in Table 1. Each object was observed at least two times and line fluxes were derived from the average of all measures for the same object.

Data reduction was performed using the IRAF package, following the standard procedure for longslit spectra: correction of bias, flat-field, extraction, wavelength and flux calibration. For flux calibration, we observed at least three spectrophotometric standard stars each night. Atmospheric extinction was corrected through mean coefficients derived for each observatory.

Line fluxes were calculated adopting Gaussian profiles, and a Gaussian de-blending routine was used when necessary. Line fluxes are given in Tables 2 and 3 using the usual scale of $F(\mathrm{H} \beta)=100$. The $\mathrm{H} \beta$ fluxes through the slit, in physical units $\left(\mathrm{erg} / \mathrm{cm}^{2} \mathrm{~s}\right)$, and corrected for interstellar and atmospheric reddening are listed in Table 4. Interstellar reddening was derived from the Balmer ratio $\mathrm{H} \alpha / \mathrm{H} \beta$, assuming Case B (Osterbrock 1989), using the interstellar extinction law by Cardelli et al. (1989) and adopting $R_{V}=3.1$ for all objects. $E(B-V)$ values derived for the nebulae are given in Table 4 .

All objects from Beaulieu et al. (1999) and Kohoutek's (1994) Table 5 were observed. Those not present in Table 1 do not have a $S / N$ satisfactory for plasma diagnostics. In particular, objects K5-8, SB-05, SB-27 and SB-29 do not seem to be PNe.

\subsection{Physical parameters}

Electron densities were derived from the $[\mathrm{SII}] \lambda 6716 / \lambda 6731$ ratio, while electron temperatures were derived from both [OIII] $\lambda 4363 / \lambda 5007$ and $[\mathrm{NII}] \lambda 5755 / \lambda 6584$ line ratios. When both temperatures were available, the [OIII] temperature was used to derive ionic abundances of species with higher ionisation potentials as $\mathrm{O}^{+2}, \mathrm{Ar}^{+2}, \mathrm{Ar}^{+3}, \mathrm{Ne}^{+2}$, and the [NII] temperature was used for lower potential lines such as $\mathrm{O}^{+}, \mathrm{N}^{+}, \mathrm{S}^{+}$. When only one temperature was available, it was used for all
Table 1. Log of the observations.

\begin{tabular}{|c|c|c|c|c|}
\hline $\mathrm{PNe}$ & PN G / PK & Num. & Date of Obs. & Place \\
\hline K5-1 & PK $000+04.3$ & 2 & 20-Jul.-99 & LNA \\
\hline \multirow[t]{2}{*}{ K5-3 } & PN G $002.6+05.5$ & 2 & 31-May-98 & LNA \\
\hline & & 2 & 16-Aug.-99 & $\mathrm{ESO}$ \\
\hline \multirow{2}{*}{ K5-4 } & PN G 351.9-01.9 & 2 & 31-Мay-98 & LNA \\
\hline & & 2 & 15-Aug.-99 & ESO \\
\hline $\mathrm{K} 5-5$ & $\mathrm{PN}$ G $001.5+03.6$ & 2 & 20-Jul.-99 & LNA \\
\hline K5-6 & PN G 003.6+04.9 & 2 & 20-Jul.-99 & LNA \\
\hline \multirow[t]{2}{*}{$\mathrm{K} 5-7$} & PN G $003.1+04.1$ & 3 & 19-Jul.-99 & LNA \\
\hline & & 2 & 16-Aug.-99 & ESO \\
\hline K5-9 & PK 355-01.1 & 2 & 19-Jul.-99 & LNA \\
\hline \multirow[t]{2}{*}{ K5-11 } & PK 002+02.1 & 2 & 31-Мay-98 & LNA \\
\hline & & 2 & 15-Aug.-99 & ESO \\
\hline K5-12 & PK 353-03.1 & 2 & 19-Jul.-99 & LNA \\
\hline \multirow[t]{2}{*}{ K5-13 } & PK 002+02.2 & 2 & 31-May-98 & LNA \\
\hline & & 2 & 16-Aug.-99 & ESO \\
\hline \multirow{2}{*}{ K5-14 } & PN G $003.9+2.6$ & 1 & 18-Jul.-99 & LNA \\
\hline & & 1 & 19-Jul.-99 & LNA \\
\hline \multirow[t]{2}{*}{$\mathrm{K} 5-16$} & PN G $002.8+01.8$ & 1 & 31-May-98 & LNA \\
\hline & & 2 & 18-Jul.-99 & LNA \\
\hline K5-17 & PK 004+02.2 & 2 & 18-Jul.-99 & LNA \\
\hline K5-19 & $\mathrm{PN}$ G $005.1+02.0$ & 1 & 18-Jul.-99 & LNA \\
\hline \multirow{2}{*}{ K5-20 } & PN G 356.8-03.0 & 3 & 18-Jul.-99 & LNA \\
\hline & & 2 & 16-Aug.-99 & ESO \\
\hline SB 01 & PN G 000.1-08.0 & 2 & 24-Jun.-00 & LNA \\
\hline SB 02 & PN G 000.5-05.3 & 2 & un.-00 & LNA \\
\hline SB 03 & PN G 000.7-06.1 & 2 & 23-Jun.-00 & LNA \\
\hline SB 04 & PN G 001.1-06.4 & 2 & 24-Jun.-00 & LNA \\
\hline SB 06 & PN G 001.6-05.9 & 2 & un.-00 & LNA \\
\hline SB 12 & PN G 005.4-06.1 & 2 & un.-00 & LNA \\
\hline SB 15 & PN G 009.3-06.5 & 2 & 23-Jun.-00 & LNA \\
\hline SB 17 & PN G 011.1-07.9 & 2 & ug.-99 & ESO \\
\hline \multirow[t]{2}{*}{ SB 18} & PN G 011.4-07.3 & 1 & 23-Jun.-00 & LNA \\
\hline & & 2 & 25-Jun.-00 & LNA \\
\hline SB 19 & PN G 0 & 2 & ug.-99 & ESO \\
\hline SB 20 & PN G 014.8-08.4 & 2 & 22-Jun.-00 & LNA \\
\hline SB 21 & PN G 016.0-07.6 & 2 & 16-Aug.-99 & ESO \\
\hline SB 24 & PN G 017.5-09.2 & 2 & 21-Jun.-00 & LNA \\
\hline SB 25 & PN G 341.0+09.4 & 2 & 19-Aug.-99 & ESO \\
\hline SB 26 & PN G 341.7-06.0 & 2 & 21-jun.-00 & LNA \\
\hline SB 28 & PN G 342 & 2 & Aug.-99 & $\mathrm{ESO}$ \\
\hline SB 30 & PN G 343.9-05.8 & 2 & 23-Jun.-00 & LNA \\
\hline SB 31 & PNG 347.9-06.0 & 2 & 18-Aug.-99 & ESO \\
\hline \multirow[t]{2}{*}{ SB 32} & PNG 349.7-09.1 & 2 & 20-Aug.-99 & $\mathrm{ESO}$ \\
\hline & & 1 & 21-Jun.-00 & LNA \\
\hline SB 33 & 06.3 & 2 & 24-Jun.-00 & LNA \\
\hline \multirow[t]{2}{*}{ SB 34} & PNG 351.5-06.5 & 1 & 18-Aug.-99 & $\mathrm{ESO}$ \\
\hline & & 1 & 19-Aug.-99 & ESO \\
\hline \multirow[t]{2}{*}{ SB 35} & PNG 351.7-06.6 & 2 & 20-Aug.-99 & ESO \\
\hline & & 2 & 24-Jun.-00 & LNA \\
\hline SB 37 & PNG 352.6-04.9 & 2 & 19-Aug.-99 & ESO \\
\hline SB 38 & PNG 352.7-08.4 & 2 & 22-Jun.-00 & LNA \\
\hline SB 42 & PNG 355.3-07.5 & 2 & 22-Jun.-00 & LNA \\
\hline SB 44 & PNG 356.0-07.4A & 2 & 18-Aug.-99 & ESO \\
\hline SB 50 & PNG 357.3-06.5 & 2 & 22-Jun.-00 & LNA \\
\hline SB 52 & PNG 358.3-07.3 & 2 & 22-Jun.-00 & LNA \\
\hline SB 53 & PNG 358.7-05.1 & 2 & 23-Jun.-00 & LNA \\
\hline SB 55 & PNG 359.4-08.5 & 2 & 17-Aug.-99 & ESO \\
\hline
\end{tabular}


ions. Concerning densities, when [SII] lines could not be used for diagnostics, an average value of $5000 \mathrm{~cm}^{-3}$ was adopted. Derived values are shown in Table 4. Electron densities are in units of $10^{3} \mathrm{~cm}^{-3}$ and temperatures in units of $10^{4} \mathrm{~K}$.

One of the main problems of observations towards the bulge is the high extinction, which affects mainly lower intensity lines. Lines [SII] $\lambda 6716+31$ for example, are sometimes weak, but needed to derive electron densities. Other weak lines like [SIII] $\lambda 6312$ or HeII $\lambda 5412$ are also needed to derive the appropriate ionic abundances. A more serious problem arises from [OIII] $\lambda 4363$ and [NII] $\lambda 5755$ because abundances are strongly sensitive to electron temperature. Errors in these line fluxes will contribute to the derived errors in the final abundances.

Examining the line fluxes for objects measured in both observatories, no systematic differences were found, and we plot all the data together.

As we have independent measures for each object, it was possible to estimate the errors in line intensities. For this, we plot in Fig. 1 all line fluxes for all objects using reddened values, and estimate the error for each line from its average and dispersion. From this distribution, we derived the following expression for the correlation between errors and fluxes:

$\log (\Delta F)=-5.67 \pm 0.55+(0.67 \pm 0.04) \log (F)$

Where line fluxes are represented by $F$ and their errors by $\Delta F$ (in physical units). The full line in Fig. 1 represents $\Delta F=F$.

In order to estimate the errors in abundances, each line flux had its value varied randomly 500 times within its respective error interval, as well as the colour excesses, within the interval given in Table 4 . Physical parameters and abundances were then derived for each one. A histogram was built with these values and the final adopted error for each abundance was the full width of the Gaussian profile fitted to each histogram, taken at half maximum. These errors are listed in Table 6 , together with the elemental abundances.

This process was adopted to derive errors in abundances and temperatures. For densities the value distribution is not Gaussian and errors were derived simply by averaging those derived from different measures for each object. Errors in reddening correspond to the dispersion of those calculated from each measure.

We noted from our dereddened fluxes that for some objects the $\mathrm{H} \gamma$ flux is considerably different from the recombination value. This effect is stronger for low latitude objects, indicating a probable relation with variations in the total-to-selective absorption ratio $R_{V}$ for some light paths towards the bulge. Stasińska et al. (1992) have already mentioned the possibility of different $R_{V}$ values for bulge planetary nebulae. Variations in $R_{V}$, as pointed out by Cardelli et al. (1989) can have considerable effects in the blue-UV region. As the [OIII] $\lambda 4363$ line is close to $\mathrm{H} \gamma$ and important to determine electron temperatures, it

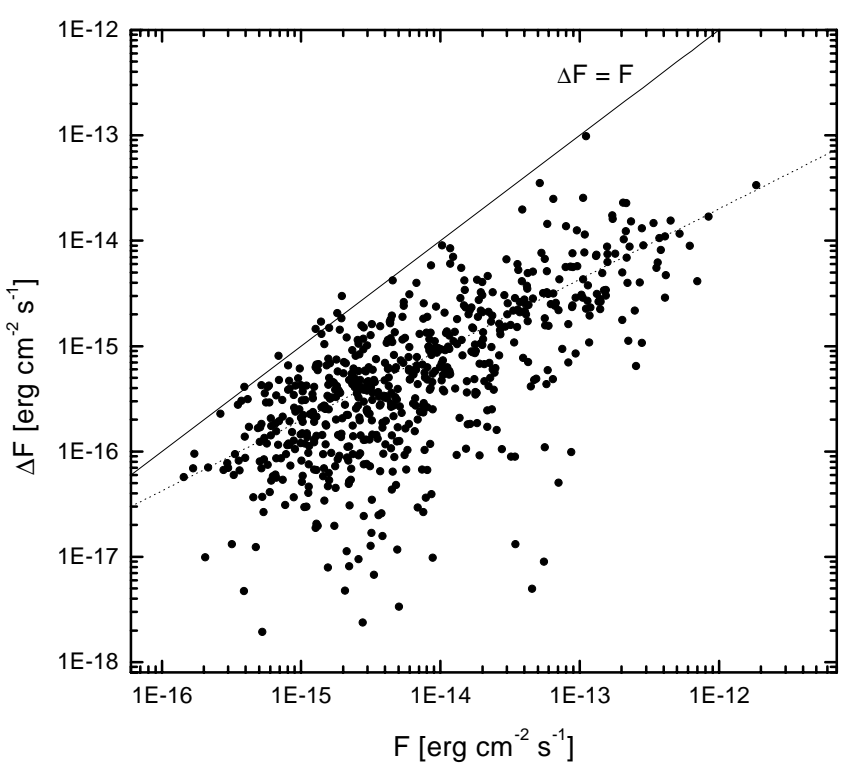

Fig. 1. Errors in line fluxes with respect to their values (in physical units).

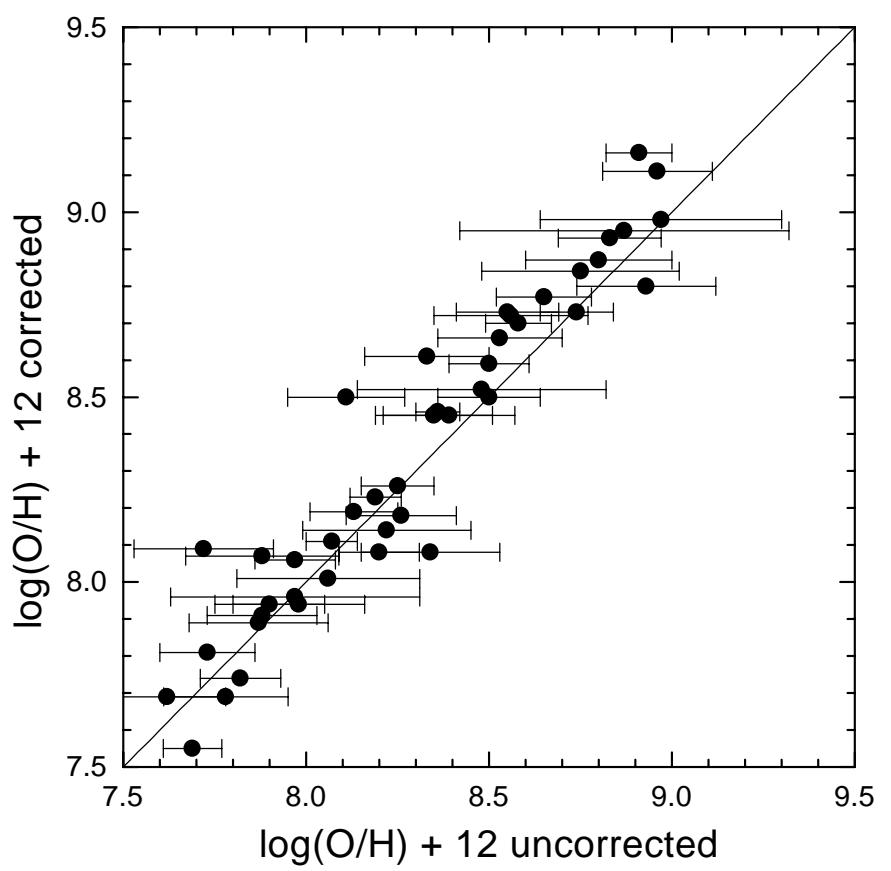

Fig. 2. Comparison between measured and rescaled oxygen abundances (see Sect. 2.2).

was necessary to check how this affects our derived physical parameters, and therefore the chemical abundances. To verify, we scaled the [OIII] $\lambda 4363$ line using the ratio between recombination and measured value for $\mathrm{H} \gamma$ as a correction factor, and rederived electron temperatures and chemical abundances for all objects. Figure 2 displays a comparison between original and rederived oxygen abundances. As can be seen, variations are within our expected errors. Chemical abundances were then rederived using these "scaled" temperatures and the procedure described below, and the results were found to be consistent with the original values within the expected errors. 
Table 4. Physical parameters.

\begin{tabular}{|c|c|c|c|c|c|c|c|c|c|}
\hline $\mathrm{PNe}$ & $E(B-V)$ & $\sigma_{E(B-V)}$ & $\log (\mathrm{H} \beta)$ & $n[\mathrm{SII}]$ & $\left.\sigma_{n[\mathrm{SII}]}\right)$ & $T[\mathrm{NII}]$ & $\sigma_{T[\mathrm{NII}]}$ & $T[\mathrm{OIII}]$ & $\sigma_{T[\mathrm{OIII}]}$ \\
\hline K 5-1 & 1.89 & 0.09 & -11.22 & 1.4 & 0.2 & 1.82 & 0.56 & 1.37 & 0.25 \\
\hline K 5-3 & 1.27 & 0.13 & -11.82 & 1.3 & 0.4 & 1.07 & 0.23 & 1.38 & 0.15 \\
\hline K 5-4 & 1.87 & 0.09 & -10.84 & 7.6 & 1.8 & 1.17 & 0.16 & 0.99 & 0.10 \\
\hline K 5-5 & 2.24 & 0.11 & -10.98 & 10.9 & - & 2.94 & 1.43 & 2.08 & 0.46 \\
\hline K 5-6 & 1.40 & 0.07 & -11.98 & 1.0 & 0.3 & - & - & 1.82 & 0.25 \\
\hline K 5-7 & 1.43 & 0.14 & -12.21 & 0.3 & 0.3 & - & - & 1.75 & 0.30 \\
\hline K 5-9 & 2.03 & 0.10 & -11.23 & 3.5 & 1.5 & 1.01 & 0.24 & - & - \\
\hline K 5-11 & 1.92 & 0.38 & -11.52 & 0.7 & 0.2 & 0.81 & 0.10 & 0.94 & 0.20 \\
\hline K 5-12 & 1.27 & 0.06 & -11.76 & 5.5 & 1.6 & 1.23 & 0.24 & 1.07 & 0.09 \\
\hline K 5-13 & 1.82 & 0.18 & -11.65 & 1.2 & 0.1 & 0.90 & 0.11 & 1.36 & 0.25 \\
\hline K 5-14 & 1.42 & 0.14 & -11.67 & 8.7 & - & 1.24 & 0.17 & 1.60 & 0.17 \\
\hline K 5-16 & 1.98 & 0.40 & -11.42 & 0.7 & 0.1 & 0.90 & 0.10 & - & - \\
\hline K 5-17 & 1.53 & 0.08 & -11.42 & 11.6 & - & - & - & 1.47 & 0.15 \\
\hline K 5-19 & 1.85 & 0.19 & -11.68 & 1.0 & - & 1.49 & 0.41 & 2.48 & 0.57 \\
\hline K 5-20 & 1.41 & 0.14 & -11.83 & 0.5 & - & - & - & 1.10 & 0.15 \\
\hline SB 01 & 0.71 & 0.07 & -12.99 & 1.4 & 1.3 & - & - & 1.33 & 0.35 \\
\hline SB 02 & 0.63 & 0.06 & -12.63 & 0.2 & 0.1 & 1.41 & 0.22 & 1.46 & 0.17 \\
\hline SB 03 & 0.52 & 0.05 & -12.67 & 0.3 & - & 1.50 & 0.26 & 3.09 & 0.38 \\
\hline SB 04 & 0.43 & 0.04 & -13.26 & 0.1 & 0.1 & - & - & 1.86 & 0.27 \\
\hline SB 06 & 0.47 & 0.05 & -13.30 & 0.1 & - & - & - & 2.20 & 0.37 \\
\hline SB 12 & 0.11 & 0.11 & -14.58 & - & - & - & - & 2.35 & 0.61 \\
\hline SB 15 & 0.42 & 0.21 & -13.07 & - & - & - & - & 2.08 & 0.23 \\
\hline SB 17 & 0.58 & 0.06 & -13.25 & 0.1 & - & 1.22 & 0.19 & - & - \\
\hline SB 18 & 0.31 & 0.09 & -13.49 & 0.3 & - & - & - & 1.82 & 0.22 \\
\hline SB 19 & 0.49 & 0.05 & -13.18 & 1.8 & - & - & - & 0.90 & 0.11 \\
\hline SB 20 & 0.40 & 0.08 & -13.27 & - & - & - & - & 2.58 & 0.29 \\
\hline SB 21 & 0.47 & 0.05 & -12.96 & - & - & - & - & 1.70 & 0.20 \\
\hline SB 24 & 0.37 & 0.02 & -13.68 & 9.1 & - & - & - & 1.45 & 0.25 \\
\hline SB 25 & 1.05 & 0.11 & -12.86 & 0.2 & 0.1 & - & - & 1.30 & 0.29 \\
\hline SB 26 & 0.60 & 0.03 & -12.70 & - & - & - & - & 1.62 & 0.16 \\
\hline SB 28 & 0.51 & 0.05 & -13.44 & - & - & - & - & 2.90 & 0.64 \\
\hline SB 30 & 0.47 & 0.02 & -12.05 & 5.5 & 2.8 & - & - & 1.42 & 0.07 \\
\hline SB 31 & 0.70 & 0.04 & -12.40 & 1.0 & 0.5 & - & - & 1.58 & 0.14 \\
\hline SB 32 & 0.18 & 0.04 & -13.70 & 0.2 & 0.2 & 1.72 & 0.40 & 1.82 & 0.25 \\
\hline SB 33 & 0.50 & 0.05 & -13.56 & 0.4 & 0.1 & 2.06 & 0.59 & 2.18 & 0.38 \\
\hline SB 34 & 0.56 & 0.11 & -13.23 & - & - & - & - & 2.39 & 0.44 \\
\hline SB 35 & 0.49 & 0.05 & -12.76 & - & - & - & - & 1.18 & 0.10 \\
\hline SB 37 & 0.95 & 0.05 & -11.91 & 0.4 & 0.1 & - & - & 1.78 & 0.13 \\
\hline SB 38 & 0.04 & 0.04 & -13.65 & - & - & - & - & 1.85 & 0.19 \\
\hline SB 42 & 0.48 & 0.02 & -12.35 & 0.1 & 0.1 & 1.31 & 0.28 & 1.24 & 0.11 \\
\hline SB 44 & 0.11 & 0.06 & -13.73 & 0.9 & 0.3 & - & - & 1.19 & 0.15 \\
\hline SB 50 & 0.43 & 0.09 & -13.12 & 0.3 & 0.3 & - & - & 1.86 & 0.21 \\
\hline SB 52 & 0.32 & 0.03 & -12.76 & 1.0 & 1.2 & 1.46 & 0.48 & 1.46 & 0.10 \\
\hline SB 53 & 0.50 & 0.05 & -12.63 & 0.7 & 0.1 & 2.53 & 0.74 & 1.74 & 0.13 \\
\hline SB 55 & 0.46 & 0.23 & -12.95 & 1.7 & - & - & - & 2.56 & 0.50 \\
\hline
\end{tabular}

\section{Chemical abundances}

Ionic abundances were calculated for the ions present in the optical spectra by solving the statistical equilibrium equations for a three-level atom model, including radiative and collisional transitions. Elemental abundances were then derived adopting ionisation correction factors (icf's) to account for unobserved ions of each element. Atomic data and icf's used in this work were the same as those adopted by Costa et al. (1996). Derived ionic abundances are listed in Table 5, and the resulting elemental abundances are given in Table 6.

Oxygen abundance requires ionic abundances of $\mathrm{O}^{+}$ and $\mathrm{O}^{++}$. While there are strong [OIII] lines at $\lambda 4959+$ $5007 \AA$, for $\mathrm{O}^{+}$we have in principle two possibilities: the blue pair $[\mathrm{OII}] \lambda 3626+29 \AA$, and the red one $[\mathrm{OII}] \lambda 7320+$ $20 \AA$. In this work we adopted the red pair due to the greater sensitivity to reddening of the blue-UV region, as 
discussed in Sect. 2.2, and due to the greater efficiency of the instrumental set in the red region. For many objects we were not able to get flux for the $\lambda 3626+29$ pair due to low $\mathrm{S} / \mathrm{N}$. In view of this fact we chose to keep the same line for all objects. Only for a few objects, as can be seen in Tables 2 and 3, do we not have the red pair and were forced to adopt the blue one to derive $\mathrm{O}^{+}$abundance.

To verify an eventual dependence of the line adopted on the $\mathrm{O}^{+}$abundance, for those objects having both blue and red lines measured we calculated the ionic abundance from both and compare them. The result is shown in Fig. 3. As can be seen, both values are perfectly compatible, and we expect no differences in the final oxygen abundance when using blue or red [OII] lines.

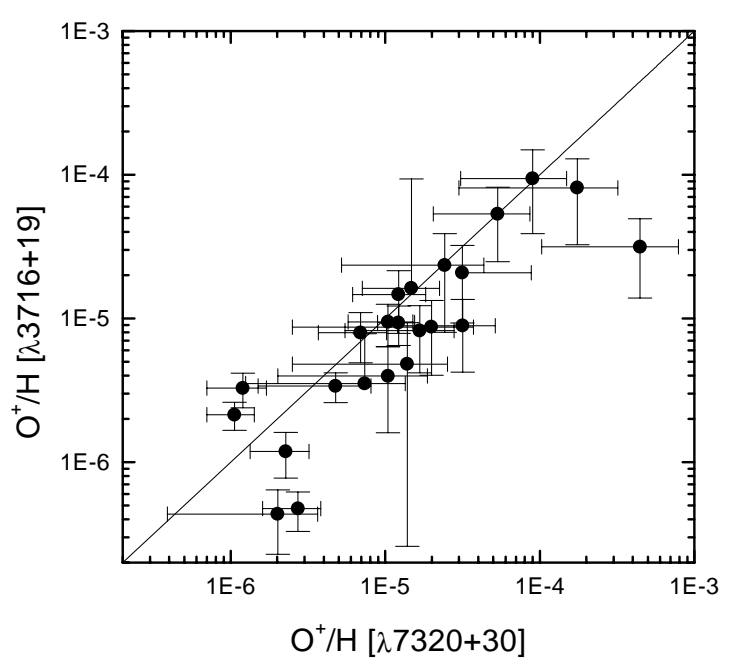

Fig. 3. $\mathrm{O}^{+}$ionic abundance derived from blue and red [OII] pairs (see text). Both values are clearly compatible, the diagonal $x=y$ line was drawn over the points and does not represent any adjustment.

Derivation of helium abundances requires special caution because collisional effects are present. In the literature there are correction factors to be applied to HeI abundances, however they may be overestimated up to a factor of two (Peimbert \& Torres-Peimbert 1987). Here we adopted the recombination theory for HeI and HeII, using recombination coefficients from Péquignot (1991). HeI abundance was also corrected for collisional effects using the correction terms from Kingdon \& Ferland (1995).

Helium abundance is given as $\mathrm{He} / \mathrm{H}$ and other elemental abundances are given as $\varepsilon(X)=\log (X / \mathrm{H})+12$. Errors in the abundances refer always to $X / \mathrm{H}$.

\section{Discussions and conclusion}

Different samples were used for this work. Most of the objects listed by Kohoutek (1994) are in the northern galactic hemisphere while those from Beaulieu et al. (1999) are mostly in the southern galactic hemisphere. Also, we selected additional data from the literature for our analysis. These additional abundances are not homogeneous with respect to observation and reduction procedures, however they are important for the sampling of the range of elemental abundances. There are few works on bulge PNe abundances, as this kind of result requires a good $\mathrm{S} / \mathrm{N}$ ratio towards a region of high extinction. Three works were then selected: Cuisinier et al. (2000), Webster (1988) and Aller \& Keyes (1987). Webster's abundances were rederived using the same icf's as we used for ours. For each of these samples we selected only objects with good spectra.

Table 6 lists our final abundances for each object. Mean values and their distribution were also derived for the sample, where distribution is represented by a $2 \sigma$ deviation from the mean value. Final values are: He: $0.126 \pm 0.054 ; \mathrm{N}: 7.64 \pm 1.10 ;$ O: $8.22 \pm 0.85 ; \mathrm{S}: 6.48 \pm 1.87$; Ar: $5.95 \pm 1.01$.

These values represent averages and FWHM of Gaussians adjusted to each histogram of abundance distribution. It should be noted that nitrogen elemental abundances depend on OII ionic abundances used as icf, so uncertainties in this last parameter will increase the dispersion found in N. As nitrogen is also a product of stellar nucleosynthesis dredged-up during the stellar evolution of the progenitors, their mass spectrum will also contribute to the derived dispersion in $\mathrm{N}$ abundance.

Sulphur abundances have to be taken cautiously because they were derived from relatively weak $[\mathrm{SII}] \lambda 6716+$ 31 and [SIII] $\lambda 6312$ lines. Besides, sulphur abundances in particular are strongly dependent on electron temperatures.

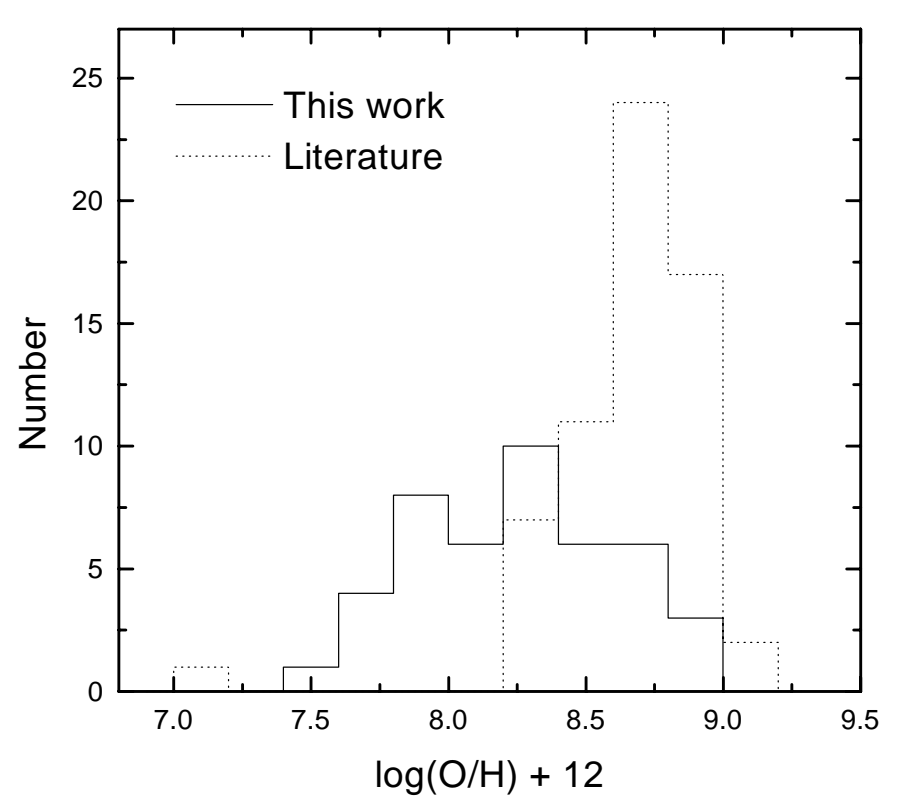

Fig. 4. Distribution of oxygen abundances for our sample (full line) and for the literature (dashed line).

Figures 4 and 5 display the abundance distribution for our sample compared to that from other works, respectively for oxygen and $\log (\mathrm{N} / \mathrm{O})$. At a first glance, distributions seem to be different, but this is due to different coverages in the sky. Our sample spans over $20 \mathrm{deg}$ in 
Table 5. Ionic abundances.

\begin{tabular}{|c|c|c|c|c|c|c|c|c|}
\hline Name & $\mathrm{HeI}$ & HeII & NII & SII & SIII & OII & OIII & ArIII \\
\hline K 5-1 & 0.108 & - & $9.29 \mathrm{E}-07$ & $2.69 \mathrm{E}-08$ & $4.57 \mathrm{E}-07$ & $2.47 \mathrm{E}-06$ & $7.29 \mathrm{E}-05$ & $5.76 \mathrm{E}-07$ \\
\hline K 5-3 & 0.082 & 0.042 & $4.19 \mathrm{E}-06$ & $1.82 \mathrm{E}-07$ & $9.44 \mathrm{E}-07$ & $3.16 \mathrm{E}-05$ & $1.17 \mathrm{E}-04$ & $4.06 \mathrm{E}-07$ \\
\hline K 5-4 & 0.109 & - & $1.02 \mathrm{E}-05$ & $3.31 \mathrm{E}-07$ & $3.97 \mathrm{E}-06$ & $5.34 \mathrm{E}-05$ & $3.91 \mathrm{E}-04$ & $1.12 \mathrm{E}-06$ \\
\hline K 5-5 & 0.03 & - & $5.63 \mathrm{E}-07$ & $4.62 \mathrm{E}-08$ & $2.93 \mathrm{E}-07$ & $2.06 \mathrm{E}-06$ & $5.08 \mathrm{E}-05$ & $2.02 \mathrm{E}-07$ \\
\hline K 5-6 & 0.023 & 0.09 & $4.06 \mathrm{E}-07$ & $4.16 \mathrm{E}-08$ & $5.59 \mathrm{E}-07$ & $2.22 \mathrm{E}-06$ & $7.07 \mathrm{E}-05$ & $2.09 \mathrm{E}-07$ \\
\hline K 5-7 & 0.066 & 0.094 & $1.25 \mathrm{E}-06$ & $7.38 \mathrm{E}-08$ & - & $5.22 \mathrm{E}-06$ & $8.44 \mathrm{E}-05$ & $6.10 \mathrm{E}-07$ \\
\hline K 5-9 & 0.142 & 0.015 & $7.73 \mathrm{E}-06$ & $2.50 \mathrm{E}-07$ & $1.29 \mathrm{E}-06$ & $4.17 \mathrm{E}-05$ & $2.32 \mathrm{E}-04$ & $1.14 \mathrm{E}-06$ \\
\hline K 5-11 & 0.174 & 0.017 & $1.13 \mathrm{E}-04$ & $2.36 \mathrm{E}-06$ & $3.19 \mathrm{E}-06$ & $4.57 \mathrm{E}-04$ & $2.22 \mathrm{E}-04$ & $1.64 \mathrm{E}-06$ \\
\hline K 5-12 & 0.046 & 0.06 & $2.91 \mathrm{E}-06$ & $2.05 \mathrm{E}-07$ & $3.11 \mathrm{E}-06$ & $1.25 \mathrm{E}-05$ & $3.45 \mathrm{E}-04$ & $5.61 \mathrm{E}-06$ \\
\hline K 5-13 & 0.082 & 0.058 & $8.68 \mathrm{E}-05$ & $2.31 \mathrm{E}-06$ & $4.20 \mathrm{E}-06$ & $1.75 \mathrm{E}-04$ & $1.95 \mathrm{E}-04$ & $1.53 \mathrm{E}-06$ \\
\hline K 5-14 & 0.094 & 0.022 & $2.50 \mathrm{E}-05$ & $8.56 \mathrm{E}-07$ & $1.68 \mathrm{E}-06$ & $8.88 \mathrm{E}-05$ & $1.71 \mathrm{E}-04$ & $9.26 \mathrm{E}-07$ \\
\hline K 5-16 & 0.129 & 0.012 & $1.28 \mathrm{E}-04$ & $4.13 \mathrm{E}-06$ & $1.78 \mathrm{E}-05$ & $4.12 \mathrm{E}-04$ & $4.47 \mathrm{E}-04$ & $2.32 \mathrm{E}-06$ \\
\hline K 5-17 & 0.076 & - & $1.77 \mathrm{E}-06$ & $1.73 \mathrm{E}-07$ & $6.41 \mathrm{E}-07$ & $6.97 \mathrm{E}-06$ & $1.52 \mathrm{E}-04$ & $3.66 \mathrm{E}-07$ \\
\hline K 5-19 & 0.071 & 0.082 & $3.34 \mathrm{E}-06$ & $1.73 \mathrm{E}-07$ & - & $7.57 \mathrm{E}-06$ & $5.29 \mathrm{E}-05$ & $2.30 \mathrm{E}-07$ \\
\hline K 5-20 & 0.128 & - & $7.30 \mathrm{E}-07$ & $3.08 \mathrm{E}-08$ & $7.78 \mathrm{E}-07$ & $3.17 \mathrm{E}-05$ & $2.14 \mathrm{E}-04$ & $4.74 \mathrm{E}-07$ \\
\hline SB 01 & 0.046 & 0.032 & $1.79 \mathrm{E}-06$ & $1.68 \mathrm{E}-07$ & - & $1.48 \mathrm{E}-05$ & $8.26 \mathrm{E}-05$ & $1.70 \mathrm{E}-07$ \\
\hline SB 02 & 0.141 & 0.027 & $6.96 \mathrm{E}-06$ & $2.48 \mathrm{E}-07$ & - & $1.99 \mathrm{E}-05$ & $5.90 \mathrm{E}-05$ & $3.62 \mathrm{E}-07$ \\
\hline SB 03 & 0.105 & 0.054 & $1.59 \mathrm{E}-06$ & $6.55 \mathrm{E}-08$ & - & $1.24 \mathrm{E}-06$ & $3.09 \mathrm{E}-05$ & $1.78 \mathrm{E}-07$ \\
\hline SB 04 & 0.095 & 0.019 & $6.34 \mathrm{E}-06$ & $1.75 \mathrm{E}-07$ & - & $6.12 \mathrm{E}-06$ & $3.86 \mathrm{E}-05$ & $1.85 \mathrm{E}-07$ \\
\hline SB 06 & 0.084 & 0.046 & $2.18 \mathrm{E}-05$ & $1.27 \mathrm{E}-07$ & - & $1.22 \mathrm{E}-05$ & $2.65 \mathrm{E}-05$ & $3.72 \mathrm{E}-07$ \\
\hline SB 12 & 0.049 & 0.066 & $2.93 \mathrm{E}-07$ & - & - & - & $4.08 \mathrm{E}-05$ & $2.52 \mathrm{E}-07$ \\
\hline SB 15 & 0.053 & 0.046 & $7.64 \mathrm{E}-08$ & $6.16 \mathrm{E}-08$ & - & $4.78 \mathrm{E}-06$ & $7.79 \mathrm{E}-05$ & $4.21 \mathrm{E}-08$ \\
\hline SB 17 & 0.142 & - & $3.06 \mathrm{E}-05$ & $1.45 \mathrm{E}-06$ & - & $8.98 \mathrm{E}-05$ & $2.58 \mathrm{E}-06$ & $5.47 \mathrm{E}-08$ \\
\hline SB 18 & 0.046 & 0.057 & $1.98 \mathrm{E}-07$ & $2.25 \mathrm{E}-08$ & - & $6.93 \mathrm{E}-06$ & $7.53 \mathrm{E}-05$ & $6.85 \mathrm{E}-08$ \\
\hline SB 19 & 0.156 & - & $2.09 \mathrm{E}-06$ & $2.48 \mathrm{E}-07$ & $5.05 \mathrm{E}-06$ & $3.16 \mathrm{E}-05$ & $3.34 \mathrm{E}-04$ & $1.85 \mathrm{E}-06$ \\
\hline SB 20 & 0.116 & 0.028 & $5.90 \mathrm{E}-08$ & $2.64 \mathrm{E}-08$ & - & $1.37 \mathrm{E}-06$ & $6.02 \mathrm{E}-05$ & $4.48 \mathrm{E}-08$ \\
\hline SB 21 & 0.021 & 0.102 & $1.02 \mathrm{E}-07$ & - & - & $3.11 \mathrm{E}-06$ & $5.04 \mathrm{E}-05$ & $2.01 \mathrm{E}-07$ \\
\hline SB 24 & 0.032 & 0.077 & $5.68 \mathrm{E}-07$ & $1.56 \mathrm{E}-07$ & - & $1.67 \mathrm{E}-05$ & $4.79 \mathrm{E}-05$ & $1.62 \mathrm{E}-07$ \\
\hline SB 25 & 0.138 & - & $3.24 \mathrm{E}-05$ & $9.02 \mathrm{E}-07$ & $8.16 \mathrm{E}-07$ & $2.94 \mathrm{E}-05$ & $8.54 \mathrm{E}-05$ & $8.10 \mathrm{E}-07$ \\
\hline SB 26 & 0.016 & 0.082 & $2.19 \mathrm{E}-07$ & - & $1.45 \mathrm{E}-07$ & $1.20 \mathrm{E}-06$ & $8.96 \mathrm{E}-05$ & $1.50 \mathrm{E}-07$ \\
\hline SB 28 & 0.004 & 0.11 & $3.86 \mathrm{E}-08$ & - & - & - & $1.84 \mathrm{E}-05$ & $5.70 \mathrm{E}-08$ \\
\hline SB 30 & 0.061 & 0.04 & $1.92 \mathrm{E}-06$ & $6.88 \mathrm{E}-08$ & $3.46 \mathrm{E}-07$ & $1.22 \mathrm{E}-05$ & $1.27 \mathrm{E}-04$ & $1.87 \mathrm{E}-07$ \\
\hline SB 31 & 0.009 & 0.101 & $3.33 \mathrm{E}-07$ & $2.17 \mathrm{E}-08$ & $8.03 \mathrm{E}-07$ & $2.27 \mathrm{E}-06$ & $6.84 \mathrm{E}-05$ & $1.59 \mathrm{E}-07$ \\
\hline SB 32 & 0.12 & 0.022 & $6.71 \mathrm{E}-06$ & $4.40 \mathrm{E}-07$ & $2.66 \mathrm{E}-06$ & $2.43 \mathrm{E}-05$ & $4.32 \mathrm{E}-05$ & $2.05 \mathrm{E}-07$ \\
\hline SB 33 & 0.079 & 0.016 & $7.51 \mathrm{E}-06$ & $3.55 \mathrm{E}-07$ & - & $1.04 \mathrm{E}-05$ & $2.43 \mathrm{E}-05$ & $2.85 \mathrm{E}-07$ \\
\hline SB 34 & 0.01 & 0.129 & $4.09 \mathrm{E}-07$ & - & - & - & $2.37 \mathrm{E}-05$ & $7.48 \mathrm{E}-08$ \\
\hline SB 35 & 0.107 & - & $6.09 \mathrm{E}-07$ & $4.24 \mathrm{E}-08$ & - & $1.04 \mathrm{E}-05$ & $1.67 \mathrm{E}-04$ & $3.87 \mathrm{E}-07$ \\
\hline SB 37 & - & 0.146 & $7.93 \mathrm{E}-07$ & $4.91 \mathrm{E}-08$ & $1.13 \mathrm{E}-06$ & $1.06 \mathrm{E}-06$ & $9.73 \mathrm{E}-05$ & - \\
\hline SB 38 & 0.077 & 0.058 & $1.24 \mathrm{E}-07$ & $3.83 \mathrm{E}-08$ & - & $4.76 \mathrm{E}-07$ & $6.68 \mathrm{E}-05$ & $1.40 \mathrm{E}-07$ \\
\hline SB 42 & 0.051 & 0.004 & $1.08 \mathrm{E}-06$ & $4.22 \mathrm{E}-08$ & - & $1.39 \mathrm{E}-05$ & $4.70 \mathrm{E}-05$ & $1.52 \mathrm{E}-07$ \\
\hline SB 44 & 0.029 & 0.107 & $3.25 \mathrm{E}-06$ & $1.39 \mathrm{E}-07$ & - & $5.30 \mathrm{E}-05$ & $1.27 \mathrm{E}-04$ & $6.99 \mathrm{E}-07$ \\
\hline SB 50 & 0.056 & 0.086 & $7.41 \mathrm{E}-07$ & $8.29 \mathrm{E}-08$ & - & $3.56 \mathrm{E}-06$ & $5.02 \mathrm{E}-05$ & $2.73 \mathrm{E}-07$ \\
\hline SB 52 & 0.033 & 0.103 & $3.93 \mathrm{E}-07$ & $5.52 \mathrm{E}-08$ & $6.56 \mathrm{E}-07$ & 7.37E-06 & $8.44 \mathrm{E}-05$ & $4.09 \mathrm{E}-07$ \\
\hline SB 53 & 0.015 & 0.119 & $5.56 \mathrm{E}-07$ & $3.81 \mathrm{E}-08$ & $1.45 \mathrm{E}-06$ & $1.10 \mathrm{E}-06$ & $7.61 \mathrm{E}-05$ & $4.75 \mathrm{E}-07$ \\
\hline SB 55 & 0.035 & 0.118 & $1.08 \mathrm{E}-07$ & $3.64 \mathrm{E}-09$ & - & $2.02 \mathrm{E}-06$ & $1.50 \mathrm{E}-05$ & $8.31 \mathrm{E}-08$ \\
\hline
\end{tabular}

galactic longitude and $11 \mathrm{deg}$ in latitude, in a nearly homogeneous distribution, while data from the literature are strongly concentrated nearer the position of the galactic center. These different distributions can be seen in Fig. 6, where both samples are mapped in galactic co-ordinates.

$\mathrm{N} / \mathrm{O}$ distribution reflects the mass distribution of the progenitors, with more massive objects having higher $\mathrm{N}$ abundances. A few objects with $\log (\mathrm{N} / \mathrm{O})<-1$ could be originated from old, small-mass progenitors. The distribution peaks for typical type-II PNe abundances.
Concerning abundances, Fig. 7 displays the oxygen abundance distribution in latitude for our sample, compared with results from the literature taken from the three works mentioned before. Examining the results, we see in our sample a concentration of objects around $\varepsilon(\mathrm{O})=8.0$ at latitudes greater than $5 \mathrm{deg}$, which deviates from the average distribution. These objects belong to a region where no PNe abundances have been determined before. They can be thick disk objects seen towards the bulge, or effectively bulge objects at higher altitudes above the 
Table 6. Elemental abundances.

\begin{tabular}{|c|c|c|c|c|c|c|c|c|c|c|c|}
\hline Planetary & $\mathrm{He}$ & $\sigma_{\mathrm{He}}$ & $\varepsilon(\mathrm{N})$ & $\sigma_{\mathrm{N}}$ & $\varepsilon(\mathrm{S})$ & $\sigma_{\mathrm{S}}$ & $\varepsilon(\mathrm{O})$ & $\sigma_{\mathrm{O}}$ & $\varepsilon(\mathrm{Ar})$ & $\sigma_{\mathrm{Ar}}$ & Notes \\
\hline K 5-1 & 0.108 & 0.031 & 7.45 & 0.30 & 6.91 & 0.68 & 7.88 & 0.21 & 5.9 & 0.16 & \\
\hline K 5-3 & 0.123 & 0.019 & 7.48 & 0.20 & 6.45 & 0.27 & 8.35 & 0.16 & 6.02 & 0.17 & \\
\hline K 5-4 & 0.109 & 0.014 & 7.93 & 0.17 & 7.24 & 0.28 & 8.65 & 0.13 & 6.23 & 0.14 & \\
\hline K 5-5 & 0.030 & 0.013 & 7.16 & 0.24 & 6.67 & 0.48 & 7.72 & 0.19 & 5.45 & 0.17 & 1 \\
\hline K 5-6 & 0.114 & 0.017 & 7.81 & 0.21 & 7.05 & 0.18 & 8.55 & 0.14 & 6.15 & 0.15 & \\
\hline K 5-7 & 0.160 & 0.031 & 7.72 & 0.14 & 5.8 & 0.17 & 8.33 & 0.17 & 6.32 & 0.18 & \\
\hline K 5-9 & 0.157 & 0.030 & 7.75 & 0.15 & 6.7 & 0.31 & 8.48 & 0.34 & 6.3 & 0.26 & \\
\hline K 5-11 & 0.191 & 0.056 & 8.26 & 0.22 & 6.92 & 0.38 & 8.87 & 0.45 & 6.87 & 0.67 & \\
\hline K 5-12 & 0.106 & 0.013 & 8.28 & 0.28 & 7.71 & 0.51 & 8.91 & 0.09 & 7.25 & 0.10 & \\
\hline K 5-13 & 0.140 & 0.029 & 8.5 & 0.11 & 7.03 & 0.20 & 8.8 & 0.20 & 6.82 & 0.27 & \\
\hline K 5-14 & 0.116 & 0.019 & 7.95 & 0.11 & 6.68 & 0.15 & 8.5 & 0.14 & 6.37 & 0.22 & \\
\hline K 5-16 & 0.141 & 0.042 & 8.47 & 0.09 & 7.56 & 0.31 & 8.97 & 0.33 & 6.82 & 0.64 & \\
\hline K 5-17 & 0.076 & 0.015 & 7.61 & 0.17 & 6.99 & 0.25 & 8.2 & 0.11 & 5.71 & 0.12 & \\
\hline K 5-19 & 0.153 & 0.031 & 7.76 & 0.25 & 5.82 & 0.20 & 8.11 & 0.16 & 5.88 & 0.22 & \\
\hline K 5-20 & 0.128 & 0.027 & 6.75 & 0.13 & 6.48 & 0.21 & 8.39 & 0.18 & 5.86 & 0.19 & \\
\hline SB 01 & 0.077 & 0.016 & 7.3 & 0.16 & 5.74 & 0.16 & 8.22 & 0.23 & 5.66 & 0.19 & 1 \\
\hline SB 02 & 0.168 & 0.024 & 7.52 & 0.13 & 5.74 & 0.09 & 7.97 & 0.11 & 5.89 & 0.12 & \\
\hline SB 03 & 0.159 & 0.020 & 7.8 & 0.11 & 5.96 & 0.1 & 7.69 & 0.08 & 5.58 & 0.09 & 2,3 \\
\hline SB 04 & 0.114 & 0.028 & 7.75 & 0.12 & 5.79 & 0.11 & 7.73 & 0.13 & 5.54 & 0.13 & \\
\hline SB 06 & 0.130 & 0.029 & 8.03 & 0.09 & 5.4 & 0.13 & 7.78 & 0.17 & 6.05 & 0.15 & \\
\hline SB 12 & 0.115 & 0.034 & - & - & - & - & 7.98 & 0.18 & 5.9 & 0.25 & \\
\hline SB 15 & 0.099 & 0.017 & 6.39 & 0.17 & 5.72 & 0.13 & 8.19 & 0.07 & 5.05 & 0.19 & \\
\hline SB 17 & 0.142 & 0.033 & 7.5 & 0.15 & 6.32 & 0.16 & 7.97 & 0.34 & 6.42 & 0.35 & \\
\hline SB 18 & 0.103 & 0.023 & 6.72 & 0.26 & 5.11 & 0.23 & 8.26 & 0.15 & 5.35 & 0.21 & \\
\hline SB 19 & 0.156 & 0.031 & 7.38 & 0.19 & 7.47 & 0.28 & 8.56 & 0.21 & 6.43 & 0.16 & 3 \\
\hline SB 20 & 0.144 & 0.029 & 6.52 & 0.17 & 5.85 & 0.18 & 7.88 & 0.15 & 4.88 & 0.19 & 3 \\
\hline SB 21 & 0.123 & 0.017 & 7.02 & 0.16 & - & - & 8.5 & 0.11 & 6.23 & 0.13 & \\
\hline SB 24 & 0.109 & 0.018 & 6.87 & 0.30 & 5.53 & 0.37 & 8.34 & 0.19 & 6 & 0.21 & \\
\hline SB 25 & 0.138 & 0.038 & 8.1 & 0.15 & 6.58 & 0.22 & 8.06 & 0.25 & 6.16 & 0.21 & 3 \\
\hline SB 26 & 0.098 & 0.013 & 8 & 0.17 & 6.88 & 0.26 & 8.74 & 0.10 & 6.09 & 0.12 & \\
\hline SB 28 & 0.114 & 0.021 & - & - & - & - & 8.75 & 0.27 & 6.37 & 0.28 & \\
\hline SB 30 & 0.100 & 0.010 & 7.56 & 0.12 & 6.35 & 0.17 & 8.36 & 0.06 & 5.66 & 0.06 & \\
\hline SB 31 & 0.110 & 0.011 & 8.13 & 0.20 & 7.15 & 0.19 & 8.96 & 0.15 & 6.45 & 0.14 & \\
\hline SB 32 & 0.142 & 0.032 & 7.34 & 0.14 & 6.76 & 0.20 & 7.9 & 0.15 & 5.71 & 0.16 & \\
\hline SB 33 & 0.095 & 0.029 & 7.48 & 0.13 & 5.85 & 0.12 & 7.62 & 0.16 & 5.82 & 0.16 & \\
\hline SB 34 & 0.139 & 0.023 & - & - & - & - & 8.53 & 0.17 & 6.16 & 0.19 & \\
\hline SB 35 & 0.107 & 0.016 & 7.02 & 0.12 & 5.55 & 0.15 & 8.25 & 0.10 & 5.74 & 0.11 & \\
\hline SB 37 & 0.146 & 0.015 & - & - & 7.91 & 0.17 & - & - & - & - & \\
\hline SB 38 & 0.135 & 0.017 & 7.49 & 0.16 & 6.65 & 0.19 & 8.07 & 0.07 & 5.52 & 0.12 & 3 \\
\hline SB 42 & 0.055 & 0.010 & 6.71 & 0.19 & 5 & 0.10 & 7.82 & 0.11 & 5.45 & 0.11 & \\
\hline SB 44 & 0.136 & 0.017 & 7.72 & 0.18 & 5.45 & 0.17 & 8.93 & 0.19 & 6.8 & 0.18 & \\
\hline SB 50 & 0.141 & 0.021 & 7.45 & 0.15 & 5.79 & 0.17 & 8.13 & 0.12 & 6 & 0.12 & 3 \\
\hline SB 52 & 0.137 & 0.011 & 7.31 & 0.34 & 6.63 & 0.59 & 8.58 & 0.09 & 6.39 & 0.10 & \\
\hline SB 53 & 0.134 & 0.012 & 8.53 & 0.2 & 7.85 & 0.4 & 8.83 & 0.14 & 6.75 & 0.12 & 3 \\
\hline SB 55 & 0.153 & 0.026 & 6.6 & 0.32 & 4.17 & 0.40 & 7.87 & 0.19 & 5.74 & 0.24 & \\
\hline
\end{tabular}

1 Spectra with large errors.

2 An average temperature was used.

3 Lines [OII] $\lambda 3726+29$ were used to derive OII abundance.

galactic plane. If their membership is confirmed, their lower abundances suggest the existence of a vertical abundance gradient within the bulge.

Figure 8 shows $\log (\mathrm{N} / \mathrm{O})$ versus helium abundance for our sample and for the same literature objects used above. As nitrogen and helium are nucleosynthesis products and related to the mass spectrum of the progenitors, they are expected to be correlated. In the figure there is an upper group of objects that, in spite of the expected dispersion due to the mass distribution of the progenitors, shows a correlation. It can also be seen that there is another small group with low $\mathrm{N} / \mathrm{O}$ and $\mathrm{He} / \mathrm{H}$ varying from 0.10 to 0.16 . The same pattern appears in the results of Cuisinier et al. (1996) and can be related to objects at high $z$ above the 


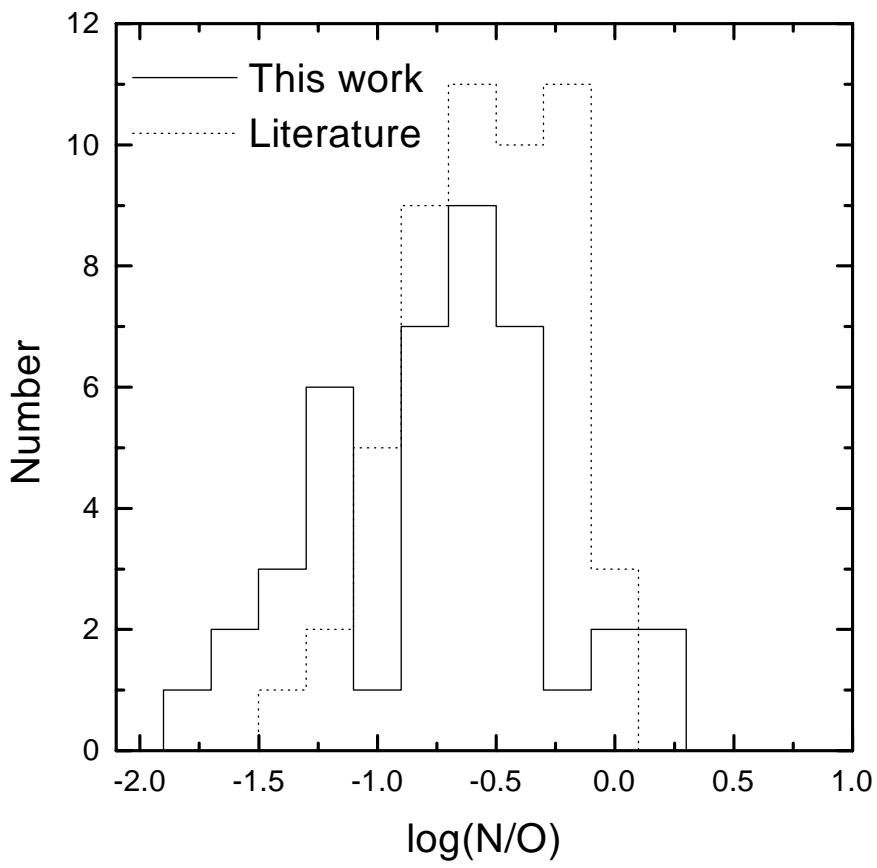

Fig. 5. Distribution of $\log (\mathrm{N} / \mathrm{O})$ for our sample (full line) and for the literature (dashed line).

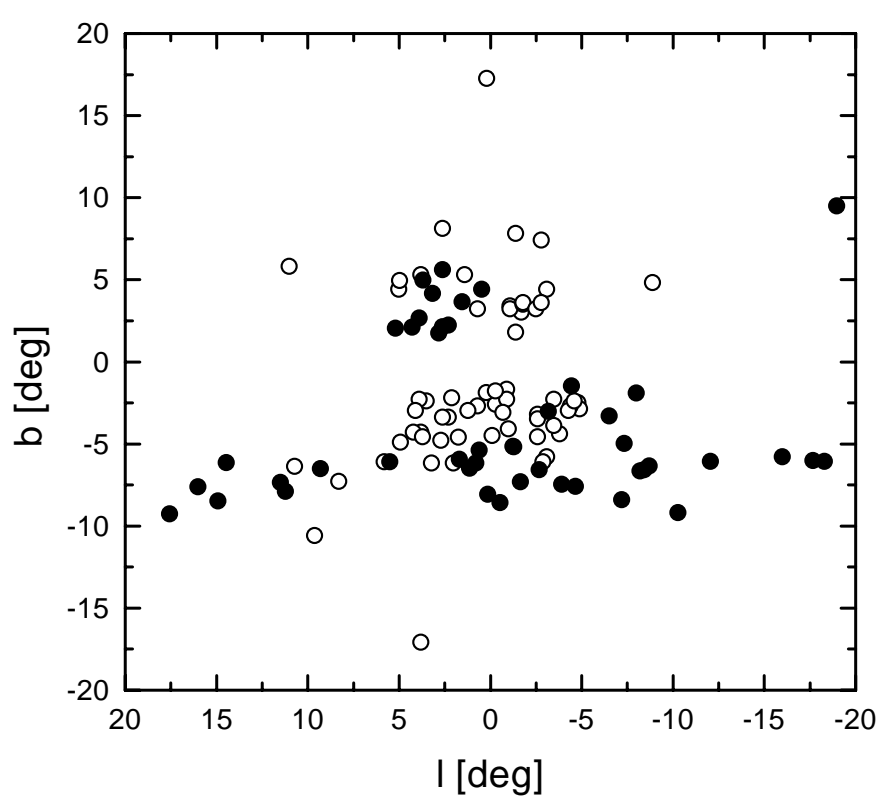

Fig. 6. Space distribution for our sample (filled circles) and for the literature (open circles).

galactic plane. However, as distances for our these object are highly uncertain, this is still an open question.

One has to take into account that bulge membership is an additional problem hard to solve for PNe. Objects from Beaulieu et al. (1999) are distributed over a wide range of galactic latitudes and their elemental abundances are lower than those from other works. This problem will be tackled in another work, now in preparation.

Acknowledgements. A. V. E. acknowledges CAPES for his graduate fellowship. Observations at ESO/Chile were possible through FAPESP grant 98/10138-8. Comments and

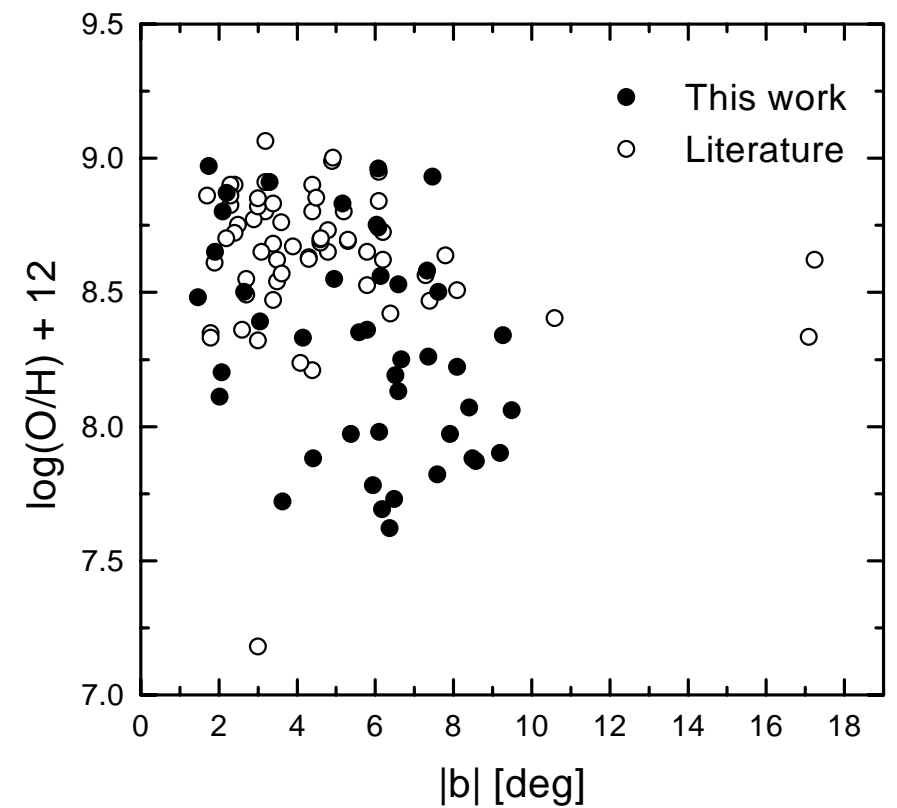

Fig. 7. Oxygen abundances vs. absolute value of galactic latitude for our sample (filled circles) and for the literature (open circles).

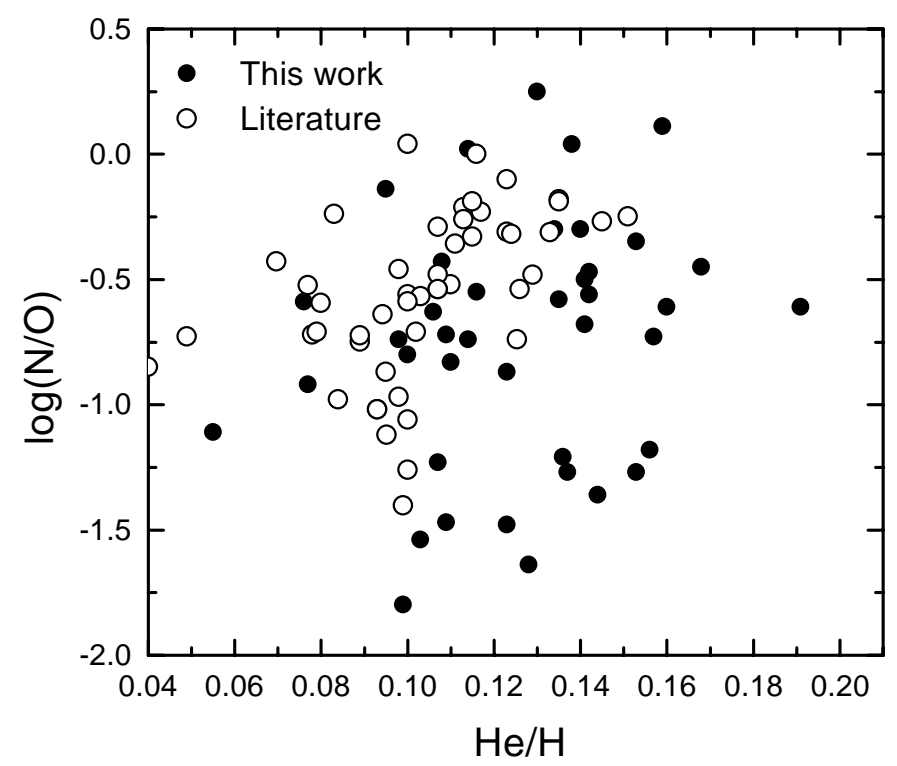

Fig. 8. $\log (\mathrm{N} / \mathrm{O})$ vs. helium abundance for our sample (filled circles) and for the literature (open circles).

suggestions of the referee helped to improve the final quality of this work.

\section{References}

Acker, A., Ochsenbein, F., Stenholm, B., et al. 1992, Strasbourg-ESO Catalogue of Galactic Planetary Nebulae Aller, L. H., \& Keyes, C. D. 1987, ApJS, 65, 405

Beaulieu, S. F., Dopita, M. A., \& Freeman, K. C. 1999, ApJ, 515,610

Cardelli, J. A., Clayton, G. C., \& Mathis, J. S. 1989, ApJ, 345, 245

Costa, R. D. D., Chiappini, C., Maciel, W. J., \& de Freitas Pacheco, J. A. 1996, A\&AS, 116, 249 
Costa, R. D. D., Idiart, T. P., \& de Freitas Pacheco, J. A. 2000, A\&AS, 145, 467

Cuisinier, F., Acker, A., \& Köppen, J. 1996, A\&A, 307, 215

Cuisinier, F., Maciel, W. J., Köppen, J., Acker, A., \& Stenholm, B. 2000, A\&A, 353, 543

Freitas Pacheco, J. A., Maciel, W. J., \& Costa, R. D. D. 1992, A\&A, 261, 579

Frogel, J. A., Terndrup, D. M., Blanco, V. M., \& Whitford, A. E. 1990, ApJ, 353, 494

Frogel, J. A., Tiede, G. P., \& Kuchinski, L. E. 1999, AJ, 117, 2296

Ibata, R. A., \& Gilmore, G. F. 1995, MNRAS, 275, 605

Idiart, T. P., de Freitas Pacheco, J. A., \& Costa, R. D. D. 1996, AJ, 111, 1169

Kingdon, J., \& Ferland, G. J. 1995, ApJ, 442, 714

Kohoutek, L. 1994, Ast. Nach., 315, 235

McWilliam, A. 1997, ARA\&A, 35, 503

McWilliam, A., \& Rich, R. M. 1994, ApJS, 91, 749
Minitti, D., Olszewski, E. W., Liebert, J., et al. 1995, MNRAS, 277,1293

Osterbrock, D. E. 1989, in Astrophysics of Gaseous Nebulae and Active Galactic Nuclei, University Science Books

Peimbert, M., \& Torres-Peimbert, S. 1987, RMxAA, 14, 540

Péquignot, D., Petitjean, P., \& Boisson, C. 1991, A\&A, 251, 680

Rich, R. M. 1988, AJ, 95, 82

Ratag, M. A., Pottasch, S. R., Dennefeld, M., \& Menzies, J. W. 1992, A\&A, 255, 255

Ratag, M. A., Pottasch, S. R., Dennefeld, M., \& Menzies, J. W. 1997, A\&AS, 126, 297

Stasińska, G., Tylenda, R., Acker, A., \& Stenholm, B. 1992, A\&A, 266, 486

Tiede, G. M., Frogel, J. A., \& Terndrup, D. M. 1995, AJ, 110, 2788

van den Bergh, S. 1996, PASP, 108, 1091

Webster, L. 1988, MNRAS, 230, 377 